\title{
Microfluidic technologies: lifting the veil of exosomes
}

\section{Editorial}

Exosomes are cell-released small membrane vesicles derived from the endolysosomal pathway with a size range of $30-150 \mathrm{~nm}$. Since the first discovery in 1981, exosomes have been found to be released from various cell types and present in many biological fluids, including blood, urine, cerebrospinal fluid and ascites. ${ }^{1}$ significant attention has been focused on exosome molecular components (e.g. proteins, mRNA and miRNA) which have been implicated in a variety of physiological functions and pathological disease states. ${ }^{2}$ Exosomes release is exacerbated in tumor cells and enriched with a group of tumor antigens, as evidenced by increased presence in plasma and ascites patients in variable cancers. ${ }^{3}$ Probing the proteomic components of circulating exosomes is an emerging paradigm for non-invasive cancer diagnosis and monitoring of treatment.

Despite the biological and medical significance, progress in exosome analysis has been slow. The molecular mechanism of exosome biogenesis is still not well understood. Extracellular vesicles (EVs) are composed of both exosomes and microvesicles (MVs) budded directly from plasma membrane (Figure 1). ${ }^{4}$ Defining exosomes is complicated by the presence of other membrane derived subcellular structures, such as apoptotic vesicles, exosome-like vesicles, membrane particles, and ectosomes. ${ }^{5}$ Due to the substantial size overlap among these membrane vesicles, confusion on the origin and nomenclature of EVs has spread through the literature. ${ }^{4}$ Molecularly differentiating exosomes from other microvesicles is absolutely needed for advancing exosome research. Current exosome purification methods; including ultracentrifugation and sucrose gradient ultra-centrifugation; are tedious, time-consuming $(>10 \mathrm{~h})$ and unable to completely discriminate exosomes with MVs. ${ }^{6}$ Most importantly, differential ultracentrifugation co-purifies multiple EVs which are secreted by different intracellular mechanisms, and thus could potentially mask disease-related biosignatures. ${ }^{7}$ Conventional filtration has been used for isolating microvesicles in uniform size of less than $150 \mathrm{~nm}$. However, the forced filtration and shearing force may cause membrane fusion and the loss of the integrity of vesicles. ${ }^{8}$ Molecular analysis of isolated microvesicles is primarily performed using Western blot, ELISA and mass spectrometry, which require lengthy processes and concentrated exosome samples (purified from more than $3 \mathrm{~mL}$ plasma or $300 \mathrm{~mL}$ cell culture media), limiting the progress in clinical investigation and utilities of exosomes. For quantitative measurement of exosome size and concentration nanoparticle tracking analysis (NTA) requires large amount (on the order of $\mathrm{mL})$ of purified and concentrated vesicles $\left(\sim 109 \mathrm{~mL}^{-1}\right)$ and is less reliable for analyzing polydisperse system. ${ }^{9}$ Flow cytometry analyzes small particles, but limited for quantitative measurement of particles below $300 \mathrm{~nm}$. Transmission electron microscopy (TEM) and atomic force microscopy (AFM) have been commonly utilized for investigating exosome morphology and size distribution recently. The microscopic protocols do not lend themselves to the highthroughput and rapid measurement. Although attempts have been made to standardize isolation and molecular detection of exosomes and microvesicles, to date, no well-defined protocols and markers for quantitative evaluation of extracellular vesicle proteins have been developed. Presently, improving and standardizing approaches for exosomes isolation and proteomic analysis is urgently needed.
Volume I Issue 3 - 2014

Zheng Zhao, Mei He

Department of Biological and Agricultural Engineering, Kansas State University, USA

Correspondence: Mei He, Department of Biological and Agricultural Engineering, College of Engineering, Kansas State University, USA, Tel +9133077383, Email meih@ksu.edu

Received: July 02, 2014 | Published: July 03, 2014

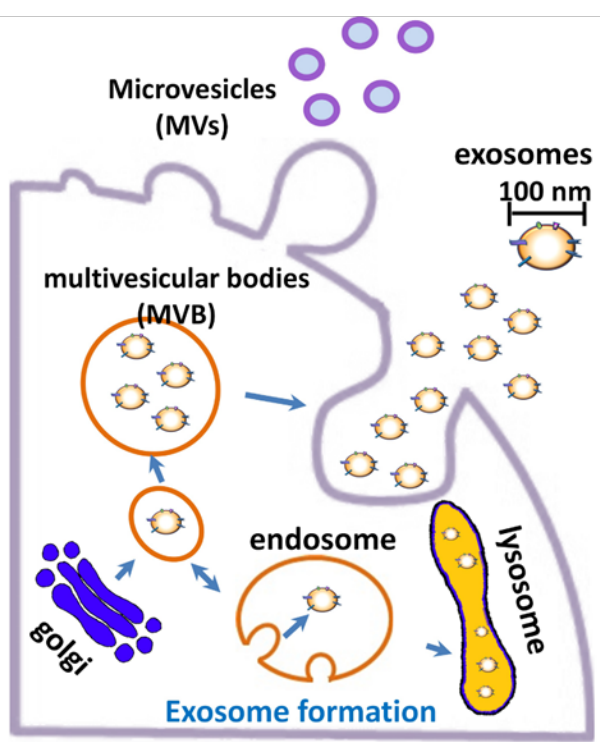

Figure I The illustration of Exosome biogenesis occurred within MVBs of the endosomal system.

The cutting-edge microfluidic technology has shown unique advantages in proteomic analysis and quantitative biology, which provides the capability of high throughput, single molecule/cell sensitivity and resolution, multifunctional integration, and automated operation with minimum sample consumption. ${ }^{10}$ Microfluidic technology has made tremendous impact on many biological and medical applications, but employing for exosome research is still in relatively early stage. Several pioneer microfluidic approaches have made enormous impact on the exosome research. A microfluidic isolation system has been developed by Chen et al. for affinity capture of serum microvesicles using surface-immobilized CD63 antibody in a straight flow micro channel. ${ }^{11}$ In contrast to targeting the surface biomarkers of microvesicles, a monolithic porous polymer membrane (100-nm size cutoff) has been integrated into micro device with an open cross flow channel for physical purification of exosomes from whole blood. ${ }^{12}$ These two microfluidic systems demonstrated superior isolation performance as opposed to the ultracentrifugation-based methods. Shailenderel al. further integrated micro channel surface capture step with the DiO-staining of exosomes in a single device, allowing simultaneous exosome isolation and quantification. ${ }^{13}$ Recently Shao et al., ${ }^{14}$ presented the first demonstration of microfluidic molecular analysis of microvesicles using a miniaturized nuclear magnetic resonance ( $\mu \mathrm{NMR})$ sensor. The immuno magnetic 
tagging-based $\mu$ NMR system was able to detect the expressions of several surface markers (e.g. EGFR, HSP90, CD41, MHCII) from purified exosomes and showed the ability to detect specific circulating microvesicles from blood of patients. This work has implied the high potential of exosomes for tracking and monitoring disease status and therapy. The further improvement has been reported by using a nanoplasmonic sensor which enables label-free detection of immunocaptured exosomes, in comparison to previous immunomagnetic tagging approach. ${ }^{15}$ The above referenced examples demonstrate the unmatched capabilities of microfluidic technology to advance molecular characterization of exosomes.

To gain better understanding of exosomes and decipher exosomal components quantitatively, it is imperative to develop new capabilities to enable comprehensive characterization of both surface and intra vesicular composition. To the best of our knowledge, few or no study has been conducted for intra vesicular proteomic analysis. It is known that exosomes transport mRNAs, microRNAs and proteins, which might be used to identify the cellular origin and can be exploited as surrogate markers of tumor. ${ }^{16}$ The linkage of differential expression of exosomal RNA content with exosome subpopulations defined by their particular surface protein topography will be the key, subsequently, for identifying the specific association of exosomes with cancer phenotypes. We anticipate that microfluidic technology could play a game-changing role in exosome research.

\section{Acknowledgements}

None.

\section{Conflict of interest}

The author declares no conflict of interest.

\section{References}

1. Keller S, Ridinger J, Rupp AK, et al. Body fluid derived exosomes as a novel template for clinical diagnostics. $J$ Transl Med. 2011;9:86.

2. Thery C, Zitvogel L, Amigorena S. Exosomes: composition, biogenesis and function. Nat Rev Immunol. 2002;2(8):569-579.

3. Skog J, Wurdinger T, van Rijn S, et al. Glioblastoma microvesicles transport RNA and proteins that promote tumour growth and provide diagnostic biomarkers. Nat Cell Biol. 2008;10(12):1470-1476.
4. Raposo G, Stoorvogel W. Extracellular vesicles: exosomes, microvesicles, and friends. J Cell Biol. 2013;200(4):373-383.

5. Akers JC, Gonda D, Kim R, et al. Biogenesis of extracellular vesicles (EV): exosomes, microvesicles, retrovirus- like vesicles, and apoptotic bodies. J Neurooncol. 2013;113(1):1-11.

6. Taylor DD, Zacharias W, Gercel-Taylor C. Exosome isolation for proteomic analyses and RNA profiling. Methods Mol Biol. 2011;728:235246.

7. Bobrie A, Colombo M, Krumeich S, et al. Diverse subpopulations of vesicles secreted by different intracellular mechanisms are present in exosome preparations obtained by differential ultracentrifugation. $J$ Extracell Vesicles. 2012:1.

8. Gyorgy B, Modos K, Pallinger E, et al. Detection and isolation of cellderived microparticles are compromised by protein complexes due to shared biophysical parameters. Blood. 2011;117(4):e39-e48.

9. Sokolova V, Ludwig AK, Hornung S, et al. Characterisation of exosomes derived from human cells by nanoparticle tracking analysis and scanning electron microscopy. Colloids Surf B Biointerfaces. 2011;87:146-150.

10. He M, Herr AE. Automated microfluidic protein immunoblotting. Nat Protoc. 2010;5(11):1844-1856.

11. Chen C, Skog J, Hsu CH, et al. Microfluidic isolation and transcriptome analysis of serum microvesicles. Lab Chip. 2010;10(4):505-511.

12. Davies RT, Kim J, Jang SC, et al. Microfluidic filtration system to isolate extracellular vesicles from blood. Lab Chip. 2012;12(24):5202-5210.

13. Kanwar SS, Dunlay CJ, Simeonecde DM, et al. Microfluidic device (ExoChip) for on-chip isolation, quantification and characterization of circulating exosomes. Lab Chip. 2014;14(11):1891-1900.

14. Shao H, Chung J, Balaj L, et al. Protein typing of circulating microvesicle sallows real-time monitoring of glioblastoma therapy. Nat $\mathrm{Med}$. 2012;18(12):1835-1840.

15. Im H, Shao H, Park YI, et al. Label-free detection and molecular profiling of exosomes with a nano-plasmonic sensor. Nat Biotechnol. 2014;32(5):490-495.

16. Taylor DD, Gercel-Taylor C. MicroRNA signatures of tumor-derived exosomes as diagnostic biomarkers of ovarian cancer. Gynecoloncol. 2008;110(1):13-21. 